\title{
Enhanced Vascular Collateralization Through the Pancreaticoduodenal Arcade Secondary to Median Arcuate Ligament Compression of the Celiac Axis in the Setting of Pancreatic Body Adenocarcinoma: The Ideal Scenario for the Modified Appleby Procedure
}

Kathleen A. Holoyda, ${ }^{*}$ Warren R. Maley, and Charles J. Yeo

\begin{abstract}
Background: A modified Appleby procedure for pancreatic body tumors relies upon collateral vessels maintaining blood flow to the proper hepatic artery (PHA) through the pancreaticoduodenal arcade (PDA) off of the superior mesenteric artery (SMA). Compression of the celiac axis by the median arcuate ligament (MAL) promotes the expansion of collateral vessels without preoperative intervention.

Case Presentation: A 51-year-old male with asymptomatic compression of the celiac artery presented with new onset insulin-dependent diabetes mellitus. He underwent imaging that demonstrated a locally advanced pancreatic body tumor that encased the superior mesenteric vein and portal vein confluence and involved the common hepatic artery. He had an adequate response to neoadjuvant FOLFIRINOX chemotherapy and underwent an uncomplicated modified Appleby procedure with a margin negative resection. Hepatic blood flow was adequate through the PHA as a result of collateralization of blood flow through the PDA off the SMA. The enhanced collateralization appeared to have occurred secondary to compression of the celiac axis by the MAL.

Conclusions: Herein we present a unique case in which improved collateral blood flow through the PDA and the gastroduodenal artery to the PHA occurred due to celiac artery compression by the MAL. This vascular anomaly fortuitously improved the ability to achieve an $\mathrm{R} 0$ resection of a locally advanced pancreatic adenocarcinoma of the body of the pancreas by a modified Appleby procedure.
\end{abstract}

Keywords: pancreatic cancer; median arcuate ligament syndrome; neoadjuvant chemotherapy; modified Appleby procedure; hepatic artery

\section{Background}

The modified Appleby procedure involves distal pancreatectomy with en bloc splenectomy and en bloc celiac axis resection for locally advanced tumors of the body and tail of the pancreas. R0 resection can be obtained following neoadjuvant therapy and demonstrates similar survival outcomes to patients with less advanced cancer. ${ }^{1}$ The success of the surgery is depen- dent upon adequate flow to the liver through the pancreaticoduodenal arcade (PDA) from the superior mesenteric artery (SMA) to the gastroduodenal artery (GDA) and the proper hepatic artery (PHA). The original procedure as described by Appleby allowed for $\mathrm{R} 0$ resection of the stomach and its blood supply for gastric carcinoma. ${ }^{2}$ To achieve R0 resection, arterial reconstruction may be necessary. ${ }^{3}$ Some surgeons

Department of General Surgery, Thomas Jefferson University, Philadelphia, Pennsylvania.

*Address correspondence to: Kathleen A. Holoyda, MD, Department of General Surgery, Thomas Jefferson University, 1015 Walnut Street Curtis Building, Suite 620, Philadelphia, PA 19107, E-mail: kathleen.holoyda@jefferson.edu 
routinely perform preoperative embolization of the common hepatic artery (CHA) to encourage collateralization through the PDA. ${ }^{4}$

Acute angulation of the origin of the celiac artery can lead to its compression by the median arcuate ligament (MAL), resulting in postprandial epigastric pain, bloating, or nausea, known as MAL syndrome. ${ }^{5}$ Twenty-one percent of patients with radiographic evidence of the MAL syndrome exhibit no symptoms. ${ }^{6}$ The compression of the celiac axis causes decreased celiac and CHA blood flow and promotes collateralization through the PDA from the SMA in a manner similar to that of preoperative embolization of the CHA.

\section{Presentation}

A 51-year-old male presented with newly diagnosed insulin-dependent diabetes mellitus and a markedly elevated Hgb-A1C. Evaluation for this condition by computerized tomography (CT) scan revealed a central pancreas tumor encasing the superior mesenteric vein (SMV) and portal vein (PV) confluence and abutting the celiac axis (involving the $\mathrm{CHA}$ ). The patient underwent seven cycles of FOLFIRINOX chemotherapy (leucovorin, fluorouracil, irinotecan, and oxaliplatin) and repeat imaging demonstrated a $2.2 \mathrm{~cm}$ mass in the neck of the pancreas abutting the CHA without involvement of the SMA, SMV, or PV (Fig. 1). The proximal celiac artery origin was flattened toward the aorta with evidence of post-stenotic dilatation, likely secondary to compression by the MAL (Fig. 2). His laboratory values after neoadjuvant therapy and the initiation of insulin therapy were notable for a CA19-9 of $55 \mathrm{U} / \mathrm{mL}$ (down from $527 \mathrm{U} / \mathrm{mL}$ ) and a $\mathrm{Hgb}-\mathrm{A} 1 \mathrm{C}$ of $5.8 \%$ (down from $11.7 \%)$.

The patient underwent a distal pancreatectomy with en bloc splenectomy and en bloc resection of his celiac axis. Due to the compression of the celiac axis by the MAL, the patient had developed enlarged collateralization from the SMA to the GDA. There was adequate flow to the PHA through the GDA. No preoperative embolization was performed and no vascular reconstruction was necessary. ${ }^{3}$ The patient tolerated the procedure well and was discharged from the hospital on postoperative day 7 after all drains were removed. At discharge he was tolerating a regular diet and he had received his triple vaccination regimen due to his post-splenectomy status. The pathology of the surgical specimen demonstrated fibrosis with scattered microscopic foci of viable but degenerating infiltrating ductal adenocarcinoma with negative surgical margins and

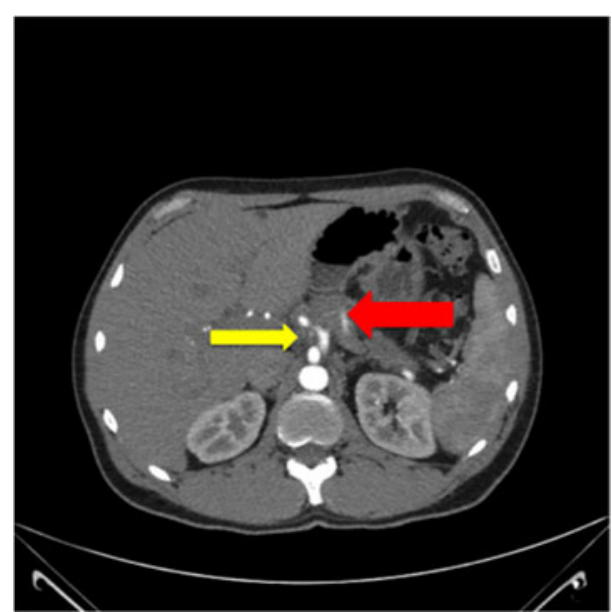

FIG. 1. Axial CT scan demonstrates close relationship of pancreatic neck and body mass to the celiac axis and CHA. Red arrow denotes hypoechoic mass. Narrow yellow arrow denotes $\mathrm{CHA}$, narrowed by the tumor. $\mathrm{CHA}$, common hepatic artery; $\mathrm{CT}$, computerized tomography.

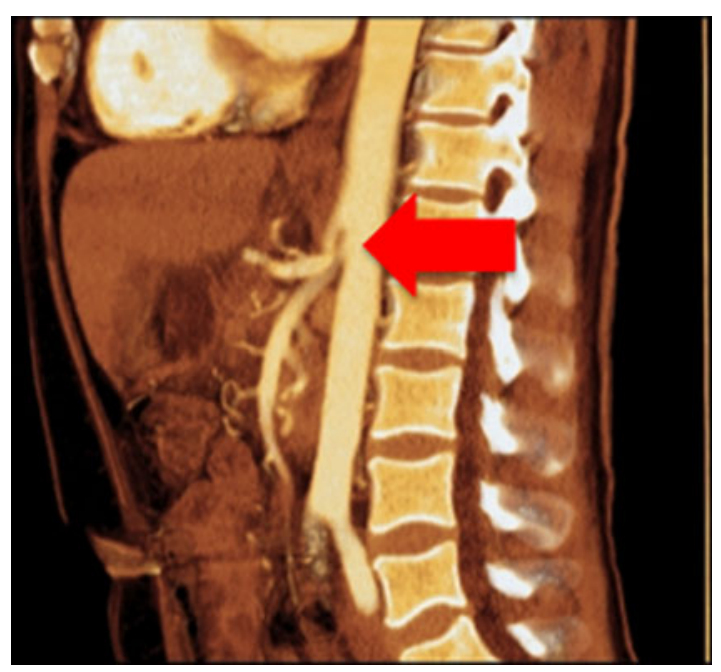

FIG. 2. Sagittal CT arteriogram reveals the acute angulation of the origin of the celiac axis off the aorta. Red arrow denotes the celiac artery origin. The MAL narrowing is depicted about $2 \mathrm{~cm}$ distal to the celiac origin. Post stenotic dilatation is seen. There was no tumor at the MAL. MAL, median arcuate ligament. 
zero out of twenty-three specimen lymph nodes involved. Two months postoperatively, the patient is doing well.

\section{Conclusions}

This is a unique case in which compression of the celiac axis by the MAL may have led to the advantageous development of enlarged collateral vessels from the SMA through the PDA. The vascular anatomy obviated the need for preoperative CHA embolization and allowed for a successful margin negative modified Appleby procedure without complication. The modified Appleby procedure can be complicated by delayed gastric emptying (20\%), partial liver infarction (6\%), and associated liver abscess $(4 \%) .{ }^{4}$ Delayed gastric emptying is believed to be secondary to ischemic gastropathy, ${ }^{7}$ and partial liver infarction and liver abscess formation occur secondary to a decrease in proper hepatic arterial pressure and blood flow, leading to necrosis of the liver with subsequent infection as a result of contaminated bile within the necrotic liver tissue. Some of these complications may be avoided with arterial resection and reconstruction in a carefully selected patient population $^{3}$ or through promotion of collateralization through preoperative embolization of the CHA. The patient presented experienced no complications and had excellent blood flow to the liver and stomach after his modified Appleby procedure. The pathology demonstrated negative resection margins and no lymph node involvement. No preoperative embolization or intraoperative vascular reconstruction was necessary as a result of the adequate collateralization that had naturally formed from the SMA secondary to his MAL compression of the celiac axis.

\section{Author Disclosure Statement}

No competing financial interests exist.

\section{References}

1. Morris M, Price T, Callahan Z, et al. Celiac axis resection with distal pancreatectomy (modified Appleby procedure) allows for R0 resection of pancreatic body and tail mass following neoadjuvant therapy: case report and literature review. Case Rep Pancreat Cancer. 2016;2:53-57.

2. Appleby LH. The coeliac axis in the expansion of the operation for gastric carcinoma. Cancer. 1953;6:704-707.

3. Latona JA, Lamb KM, Pucci MJ, et al. Modified Appleby procedure with arterial reconstruction for locally advanced pancreatic adenocarcinoma: a literature review and report of three unusual cases. J Gastrointest Surg. 2016;20:300-306.

4. Nakamura T, Hirano S, Noji T, et al. Distal pancreatectomy with en bloc celiac axis resection (modified Appleby procedure) for locally advanced pancreatic body cancer: a single-center review of 80 consecutive patients. Ann Surg Oncol. 2016;23(Suppl5):969-975.

5. Kim EN, Lamb K, Relles D, et al. Median arcuate ligament syndrome-review of this rare disease. JAMA Surg. 2016;151:471-477.

6. Derrick JR, Pollard HS, Moore RM. The pattern of arteriosclerotic narrowing of the celiac and superior mesenteric arteries. Ann Surg. 1959;149:684-689.

7. Kondo $\mathrm{S}$, Katoh $\mathrm{H}$, Hirano $\mathrm{S}$, et al. Ischemic gastropathy after distal pancreatectomy with celiac axis resection. Surg Today. 2004;34:337-340.

Cite this article as: Holoyda KA, Maley WR, Yeo CJ (2017) Enhanced vascular collateralization through the pancreaticoduodenal arcade secondary to median arcuate ligament compression of the celiac axis in the setting of pancreatic body adenocarcinoma: the ideal scenario for the modified Appleby procedure, Journal of Pancreatic Cancer 3:1, 46-48, DOI: 10.1089/pancan.2017.0008.

$\begin{aligned} & \text { Abbreviations Used } \\ \mathrm{CHA} & =\text { common hepatic artery } \\ \mathrm{GDA} & =\text { gastroduodenal artery } \\ \mathrm{MAL} & =\text { median arcuate ligament } \\ \mathrm{PDA} & =\text { pancreaticoduodenal arcade } \\ \mathrm{PHA} & =\text { proper hepatic artery } \\ \mathrm{PV} & =\text { portal vein } \\ \mathrm{SMA} & =\text { superior mesenteric artery } \\ \mathrm{SMV} & =\text { superior mesenteric vein }\end{aligned}$

